\title{
DISPLACEMENT/PRESSURE BASED MIXED FINITE ELEMENT FORMULATIONS FOR ACOUSTIC FLUID-STRUCTURE INTERACTION PROBLEMS
}

\author{
XIAODONG WANG* AND KLAUS-JÜRGEN BATHE ${ }^{\dagger}$ \\ Massachusetts Institute of Technology, Cambridge, MA 02139, U.S.A.
}

\begin{abstract}
SUMMARY
We present reliable finite element discretizations based on displacement/pressure interpolations for the analysis of acoustic fluid-structure interaction problems. The finite element interpolations are selected using the inf-sup condition, and emphasis is given to the fact that the boundary conditions must satisfy the mass and momentum conservation. We show that with our analysis procedure no spurious non-zero frequencies are encountered, as heretofore calculated with other displacement-based discretizations. (C) 1997 by John Wiley \& Sons, Ltd.
\end{abstract}

KEY WORDS: acoustics; fluid-structure interactions; mixed formulations

\section{INTRODUCTION}

Many interaction problems involving different continuous media exist in practical engineering fields. One of them is the so-called fluid-structure interaction problem where the interaction between the fluid and a structure can significantly affect the response of the structure and needs to be taken into account properly. Fluid-structure interaction problems are encountered in the analysis of offshore structures, acoustical media, liquid or gas storage tanks, pipeline systems, nuclear reactors and biomechanical systems. ${ }^{1-4}$

A number of finite element formulations have been proposed to model an acoustic fluid for the analysis of fluid-structure interaction problems, namely, the displacement formulation, ${ }^{2,5-8}$ the displacement potential and pressure formulation, and the velocity potential formulation. ${ }^{4,9-11}$

Primitive variable formulations have received considerable attention because they do not require any special interface conditions or new solution strategies (for example, in frequency calculations and response spectrum analysis). With the ever-increasing availability of high-speed and large-capacity computers, this approach shows great promise in general applications to the solution of a broad range of problems (specifically non-linear problems). Unfortunately, some difficulties have remained unsolved to use primitive variable finite element formulations in the analysis of fluid flows and fluid-structure interactions.

In linear analyses, it has been widely reported that the displacement-based fluid elements employed in frequency or dynamic analyses exhibit spurious non-zero frequency circulation modes. ${ }^{6,8,12}$ Various approaches have been introduced to obtain improved formulations. The

\footnotetext{
* Graduate Student. Presently, Assistant Professor, Institute of Paper Science \& Technology, Atlanta, GA 30318, U.S.A.

${ }^{\dagger}$ Professor of Mechanical Engineering
} 
penalty method has been applied by Hamdi et al. ${ }^{6}$ and has been shown to give good solutions for the cases considered in that reference. Subsequently, Olson and Bathe ${ }^{8}$ demonstrated that the method 'locks up' in the frequency analysis of a solid vibrating in a fluid cavity. They also showed that reduced integration performed on the penalty formulation yields some improvement in results but does not assure solution convergence in a general case. Wilson and Khalvati ${ }^{13}$ developed a formulation with rotational constraints and a reduced integration technique based on the pure displacement formulation, and Chen and Taylor ${ }^{14}$ proposed a four-node element with a reduced integration technique and an element mass matrix projection. Recently, Bermúdez and Rodríguez ${ }^{15}$ used simple three-node triangular 'edge' elements to model the fluid. This formulation is promising but the degrees of freedom of the fluid elements are not those of the structure and the coupling needs special considerations.

We believe that the currently available displacement-based formulations of fluids and fluid-structure interactions are not yet satisfactory due to lack of generality and the encountered spurious modes. There is still need to understand the nature of the spurious non-zero frequency rotational modes and obtain a general effective formulation that does not exhibit such modes.

It has been proposed that the spurious non-zero frequencies are caused by the irrotationality constraint. ${ }^{6,13,14}$ However, in this paper we show that, actually, the origins of the spurious non-zero frequencies are in the use of the pure displacement-based formulation (including the penalty formulations) and in the mishandling of the fluid-structure interface conditions. We propose displacement/pressure based formulations with proper elements (i.e. mixed elements which satisfy the inf-sup condition). In addition, we address some subtle points regarding the boundary conditions at fluid-structure interfaces. In the solutions of some selected generic test problems, we demonstrate that using the displacement/pressure formulations with proper elements and boundary conditions, we no longer encounter any spurious non-zero frequency pressure and rotational modes.

\section{UNDERSTANDING OF IRROTATIONALITY}

In linear analysis, for the isentropic and inviscid fluid models, we have the momentum and constitutive equations

$$
\begin{aligned}
\rho \ddot{\mathbf{u}}+\nabla p-\mathbf{f}^{\mathrm{B}} & =\mathbf{0} \\
\nabla \cdot \mathbf{u}+\frac{p}{\beta} & =0
\end{aligned}
$$

where $\mathbf{u}, p, \mathbf{f}^{\mathrm{B}}$ and $\beta$ are the displacement vector, pressure, body force vector and fluid bulk modulus.

If $\mathbf{f}^{\mathrm{B}}=\mathbf{0}$, then in terms of the displacements only, we have

$$
\beta \nabla(\nabla \cdot \mathbf{u})-\rho \ddot{\mathbf{u}}=\mathbf{0}
$$

Since $\nabla \times \nabla \phi=\mathbf{0}$ for any smooth scalar-valued function $\phi$, equation (3) gives

$$
\frac{\partial}{\partial t}(\nabla \times \mathbf{v})=\mathbf{0}
$$

where $\mathbf{v}$ is the velocity vector. Hence the motion is always circulation preserving, i.e. it is a motion in which the vorticity does not change with time. If the fluid starts from rest, we will have the irrotationality constraint

$$
\nabla \times \mathbf{u}=\mathbf{0}
$$


The variational form of equation (3) can be written as ${ }^{16}$

$$
\int_{V_{\mathrm{f}}}\{\beta(\nabla \cdot \mathbf{u})(\nabla \cdot \delta \mathbf{u})+\rho \ddot{\mathbf{u}} \cdot \delta \mathbf{u}\} \mathrm{d} V+\int_{S_{\mathrm{f}}} \bar{p} \delta u_{\mathrm{n}}^{S} \mathrm{~d} S=0
$$

where $u_{\mathrm{n}}^{S}$ is the displacement normal to $S_{\mathrm{f}}$. Here $V_{\mathrm{f}}, S, S_{\mathrm{f}}$ and $S_{u}$ stand for the fluid domain, fluid boundary, Neumann boundary and Dirichlet boundary, respectively. Also, we have $S_{u} \cup S_{\mathrm{f}}=S$ and $S_{u} \cap S_{\mathrm{f}}=\emptyset$.

Equation (6) is the often used pure displacement-based formulation. It was widely reported $^{6,8,12}$ that this formulation produces spurious non-zero frequency modes. From the rigid cavity test problem in Reference 6, Hamdi et al. concluded that the observed spurious non-zero frequency modes are rotational modes. They conjectured that the irrotationality constraint is 'lost' in the finite element formulation; therefore, the following penalty formulation was proposed:

$$
\int_{V_{\mathrm{f}}}\{\beta(\nabla \cdot \mathbf{u})(\nabla \cdot \delta \mathbf{u})+\alpha(\nabla \times \mathbf{u})(\nabla \times \delta \mathbf{u})+\rho \ddot{\mathbf{u}} \cdot \delta \mathbf{u}\} \mathrm{d} V+\int_{S_{\mathrm{f}}} \bar{p} \delta u_{\mathrm{n}}^{S} \mathrm{~d} S=0
$$

where $\alpha$ is a large penalty parameter.

Interestingly, for some problems, the so-called spurious non-zero frequency rotational modes disappeared with the above formulation. Considering the fact that penalty formulations are too 'stiff', researchers often use reduced integration methods for the terms

$$
\int_{V_{\mathrm{f}}}\{\beta(\nabla \cdot \mathbf{u})(\nabla \cdot \delta \mathbf{u})+\alpha(\nabla \times \mathbf{u})(\nabla \times \delta \mathbf{u})\} \mathrm{d} V
$$

It is well known now that this penalty formulation with or without reduced integration is unreliable. For example, with this method (with or without reduced integration) we cannot solve all three paradigms of fluid-structure interactions given in Section 8.3 (which were already proposed in Reference 8).

By use of reduced integration, the spurious non-zero frequency modes can be eliminated in some cases; however, a proper procedure is to replace the pure displacement-based formulation by a displacement/pressure formulation and use mixed elements that satisfy the inf-sup condition.

\section{DISPLACEMENT/PRESSURE (u/ $p$ ) FORMULATION}

We define a variational indicator

$$
\Pi=\int_{V_{\mathrm{f}}}\left\{\frac{p^{2}}{2 \beta}-\mathbf{u} \cdot \mathbf{f}^{\mathrm{B}}-\lambda_{p}\left(\frac{p}{\beta}+\nabla \cdot \mathbf{u}\right)\right\} \mathrm{d} V+\int_{S_{\mathrm{f}}} \bar{p} u_{\mathrm{n}}^{S} \mathrm{~d} S
$$

where the variables are $p$, $\mathbf{u}$, and the Lagrange multiplier $\lambda_{p}$. We note that the first two terms correspond to the usual strain energy (given in terms of the pressure) and the potential of the externally applied body forces (which may include gravity effects). The third term enforces the constitutive relation. The last term is the potential due to any applied boundary pressure on $S_{\mathrm{f}}$. To include the effects of surface gravity waves, we simply add a surface gravitational potential term $\int_{S_{\mathrm{fs}}} \frac{1}{2} \rho g u_{s}^{2} \mathrm{~d} S$, where $S_{\mathrm{fs}}$ denotes the free surface.

Invoking the stationarity of $\Pi$, we identify the Lagrange multiplier $\lambda_{p}$ to be the pressure $p$ and obtain the governing equations, with $\mathbf{f}^{\mathrm{B}}=-\rho \ddot{\mathbf{u}}$,

$$
\begin{aligned}
& \nabla p+\rho \ddot{\mathbf{u}}=\mathbf{0} \\
& \nabla \cdot \mathbf{u}+\frac{p}{\beta}=0
\end{aligned}
$$


with the boundary conditions

$$
\begin{array}{rr}
\mathbf{u} \cdot \mathbf{n}=\bar{u}_{\mathrm{n}} & \text { on } S_{u} \\
p=\bar{p} & \text { on } S_{\mathrm{f}}
\end{array}
$$

Applying the standard Galerkin discretization procedure, we have for a typical finite element

$$
\begin{aligned}
\mathbf{u} & =\mathbf{H} \hat{\mathbf{U}} \\
p & =\mathbf{H}_{p} \hat{\mathbf{P}} \\
\nabla \cdot \mathbf{u} & =(\nabla \cdot \mathbf{H}) \hat{\mathbf{U}}=\mathbf{B} \hat{\mathbf{U}}
\end{aligned}
$$

where $\mathbf{H}$ and $\mathbf{H}_{p}$ are the interpolation matrices, and $\hat{\mathbf{U}}$ and $\hat{\mathbf{P}}$ are the vectors of solution variables. ${ }^{16}$

The matrix equations of the $\mathbf{u} / p$ formulation are given as

$$
\left[\begin{array}{cc}
\mathbf{M} & \mathbf{0} \\
\mathbf{0} & \mathbf{0}
\end{array}\right]\left\{\begin{array}{l}
\ddot{\hat{\mathbf{U}}} \\
\ddot{\hat{\mathbf{P}}}
\end{array}\right\}+\left[\begin{array}{cc}
\mathbf{0} & \mathbf{L} \\
\mathbf{L}^{\mathrm{T}} & \mathbf{A}
\end{array}\right]\left\{\begin{array}{l}
\hat{\mathbf{U}} \\
\hat{\mathbf{P}}
\end{array}\right\}=\left\{\begin{array}{l}
\mathbf{R} \\
\mathbf{0}
\end{array}\right\}
$$

where

$$
\begin{aligned}
& \mathbf{M}=\int_{V_{\mathrm{f}}} \rho \mathbf{H}^{\mathrm{T}} \mathbf{H} \mathrm{d} V, \quad \mathbf{L}=-\int_{V_{\mathrm{f}}} \mathbf{B}^{\mathrm{T}} \mathbf{H}_{p} \mathrm{~d} V \\
& \mathbf{A}=-\int_{V_{\mathrm{f}}} \frac{1}{\beta} \mathbf{H}_{p}^{\mathrm{T}} \mathbf{H}_{p} \mathrm{~d} V, \quad \mathbf{R}=-\int_{S_{\mathrm{f}}} \mathbf{H}_{\mathrm{n}}^{\mathrm{S}^{\mathrm{T}}} \bar{p} \mathrm{~d} S
\end{aligned}
$$

\section{SOLVABILITY, STABILITY AND ZERO FREQUENCIES}

To analyse the solvability and stability, we define the following finite element spaces:

$$
\begin{aligned}
V_{h} & =\left\{\mathbf{v}_{h} \mid \mathbf{v}_{h}, \frac{\partial\left(v_{h}\right)_{i}}{\partial x_{j}} \in L^{2}(\mathrm{Vol}), \mathbf{v}_{h}=\mathbf{0} \text { on } S_{u}\right\} \\
D_{h} & =\left\{q_{h} \mid q_{h}=\nabla \cdot \mathbf{v}_{h} \text { for some } \mathbf{v}_{h} \in V_{h}\right\} \\
K_{h}\left(q_{h}\right) & =\left\{\mathbf{v}_{h} \mid \mathbf{v}_{h} \in V_{h}, \nabla \cdot \mathbf{v}_{h}=q_{h}\right\}
\end{aligned}
$$

where the mesh is represented by the element size $h .^{16}$

Let us now, for purposes of discussion, recall the governing algebraic finite element equations derived from the displacement/pressure formulation for incompressible (or almost incompressible) solids ${ }^{16}$

$$
\left[\begin{array}{ll}
\left(\mathbf{K}_{u u}\right)_{h} & \left(\mathbf{K}_{u p}\right)_{h} \\
\left(\mathbf{K}_{u p}\right)_{h}^{\mathrm{T}} & \left(\mathbf{K}_{p p}\right)_{h}
\end{array}\right]\left\{\begin{array}{c}
\hat{\mathbf{U}}_{h} \\
\hat{\mathbf{P}}_{h}
\end{array}\right\}=\left\{\begin{array}{c}
\mathbf{R}_{h} \\
\mathbf{0}
\end{array}\right\}
$$

where $\hat{\mathbf{U}}_{h}$ lists all the unknown nodal point displacements and $\hat{\mathbf{P}}_{h}$ lists the pressure variables. The mathematical analysis of the mixed formulation consists of a study of the solvability and the stability of equations (14); where the stability of the equations implies their solvability. ${ }^{16}$

For the corresponding dynamic problem, the governing equations are

$$
\left[\begin{array}{cc}
\left(\mathbf{M}_{u u}\right)_{h} & \mathbf{0} \\
\mathbf{0} & \mathbf{0}
\end{array}\right]\left\{\begin{array}{c}
\ddot{\hat{\mathbf{U}}}_{h} \\
\ddot{\hat{\mathbf{P}}}_{h}
\end{array}\right\}+\left[\begin{array}{cc}
\left(\mathbf{K}_{u u}\right)_{h} & \left(\mathbf{K}_{u p}\right)_{h} \\
\left(\mathbf{K}_{u p}\right)_{h}^{\mathrm{T}} & \left(\mathbf{K}_{p p}\right)_{h}
\end{array}\right]\left\{\begin{array}{c}
\hat{\mathbf{U}}_{h} \\
\hat{\mathbf{P}}_{h}
\end{array}\right\}=\left\{\begin{array}{c}
\mathbf{R}_{h} \\
\mathbf{0}
\end{array}\right\}
$$


As discussed elsewhere ${ }^{16,17}$ the key to the stability of the formulation is to satisfy the following inf-sup condition:

$$
\inf _{q_{h} \in P_{h}\left(D_{h}\right)} \sup _{\mathbf{v}_{h} \in V_{h}} \frac{\int_{V_{\mathrm{f}}} q_{h} \nabla \cdot \mathbf{v}_{h} \mathrm{~d} V_{\mathrm{f}}}{\left\|q_{h}\right\|\left\|\mathbf{v}_{h}\right\|} \geqslant \beta_{0}>0
$$

where $\beta_{0}$ is a constant independent of $h$ and the bulk modulus. If this condition is satisfied, the stability of the formulation is guaranteed and optimal error bounds are obtained for the selected displacement and pressure interpolations.

If the bulk modulus is finite, we can statically condense out (at least in theory) the pressure unknowns and have

$$
\left(\mathbf{M}_{u u}\right)_{h} \ddot{\mathbf{U}}_{h}+\left(\mathbf{K}_{u u}^{*}\right)_{h} \hat{\mathbf{U}}_{h}=\mathbf{R}_{h}
$$

where

$$
\left(\mathbf{K}_{u u}^{*}\right)_{h}=\left(\mathbf{K}_{u u}\right)_{h}-\left(\mathbf{K}_{u p}\right)_{h}\left(\mathbf{K}_{p p}^{-1}\right)_{h}\left(\mathbf{K}_{u p}\right)_{h}^{\mathbf{T}}
$$

Of course, in equation (18) the first part of the stiffness matrix is due to the deviatoric strain energy and the second part is due to the volumetric strain energy.

Considering equation (18), we have

$$
\left(\mathbf{K}_{u u}\right)_{h} \rightarrow \text { positive definite in } K_{h}\left(q_{h}\right) \quad \text { (for the shear modulus } G>0 \text { ) }
$$

where $q_{h}=\nabla \cdot \mathbf{v}_{h} \ll 1$ and

$$
\left(\mathbf{K}_{u p}\right)_{h}\left(\mathbf{K}_{p p}^{-1}\right)_{h}\left(\mathbf{K}_{u p}\right)_{h}^{\mathbf{T}} \rightarrow \operatorname{rank} m
$$

where $m$ is the number of pressure degrees of freedom (we assume that, if applicable, the physical constant pressure mode has been removed, see Reference 16, Section 4.5).

In dynamic analysis, for each time step, the coefficient matrix is given as

$$
\left(\mathbf{K}_{u u}^{* *}\right)_{h}=\left(\mathbf{K}_{u u}^{*}\right)_{h}+\mathscr{C}\left(\mathbf{M}_{u u}\right)_{h}
$$

where the constant $\mathscr{C}$ is a positive number related to the direct time integration scheme used (for instance, $\mathscr{C}=4 / \Delta t^{2}$ for the trapezoidal rule) and $\left(\mathbf{K}_{u u}^{* *}\right)_{h}$ is always a positive-definite matrix.

We can now consider the following three categories of problems:

(1) the solid bulk modulus $\kappa$ and solid shear modulus $G$ are of the same order;

(2) $\kappa \gg G$ and $\kappa, G>0$;

(3) $\kappa>0$ and $G=0$.

In category (1), the standard displacement formulation ensures the solvability and stability. In category (2), i.e. (almost) incompressible material analysis, the displacement/pressure mixed formulation with mixed elements that satisfy the inf-sup condition is well established. ${ }^{16,17}$ Category (3) includes the analysis of the inviscid acoustic fluid model discussed in this paper. In this category, the loss of ellipticity introduces zero frequency modes corresponding to the zero deviatoric strain energy (note that for this case $\left.\left(\mathbf{K}_{u u}\right)_{h}=\mathbf{0}\right)$. A mathematical prediction of the exact number of zero frequencies is necessary to identify whether or not we have non-zero frequency spurious modes. For $n$ displacement unknowns, the exact number of zero frequencies is $n-m$, provided, as mentioned above, the physical constant pressure mode arising with the boundary condition

$$
\mathbf{u} \cdot \mathbf{n}=0 \quad \text { on } S
$$


has been eliminated. Appropriate interpolations for solution are for example summarized in References 16 and 18 . The $\mathbf{u} / p$ elements correspond to continuous displacements and discontinuous pressures whereas the $\mathbf{u} / p-c$ elements yield continuous displacements and pressures across the element boundaries.

For example, in two-dimensional analysis, two effective elements are the $9 / 3$ and $9 / 4-c$ elements that are schematically depicted in Figure 1 . For the $9 / 3$ element, we interpolate the pressure linearly as

$$
p=p_{1}+p_{2} r+p_{3} s
$$

and for the $9 / 4-c$ element we use the bilinear interpolation

$$
p=p_{1}+p_{2} r+p_{3} s+p_{4} r s
$$

If we are to use four-node quadrilateral mixed elements in general meshes, the element proposed in Reference 19 is the only one known to satisfy the inf-sup condition. The well-known $4 / 1(\mathrm{Q} 1 / \mathrm{P} 0)$ element is not recommended for use in general meshes because it does not satisfy the inf-sup condition ${ }^{16}$ (but can be employed in special mesh configurations ${ }^{20}$ ).

\section{5. $\mathbf{u}-p-\boldsymbol{\Lambda}$ FORMULATION}

We note that in the $\mathbf{u} / p$ formulation, equation (5) is not imposed and that the solution gives many exact zero frequencies. In order to reduce the number of zero frequency modes, the constraint of equation (5) can be imposed by use of

$$
\nabla \times \mathbf{u}=\Lambda / \alpha
$$

where $\Lambda$ is a 'vorticity moment' of small magnitude and $\alpha$ is a constant of large value.

This constraint was used in Reference 21 , where the $\mathbf{u}-p-\boldsymbol{\Lambda}$ formulation was proposed. We want to review this formulation here briefly and then show the relationship to the $\mathbf{u} / p$ formulation.

The variational indicator in the $\mathbf{u}-p-\boldsymbol{\Lambda}$ formulation is

$$
\begin{aligned}
\Pi= & \int_{V_{\mathrm{f}}}\left\{\frac{p^{2}}{2 \beta}-\mathbf{u} \cdot \mathbf{f}^{\mathrm{B}}-\lambda_{p}\left(\frac{p}{\beta}+\nabla \cdot \mathbf{u}\right)+\frac{\boldsymbol{\Lambda} \cdot \boldsymbol{\Lambda}}{2 \alpha}-\lambda_{\boldsymbol{\Lambda}} \cdot\left(\frac{\boldsymbol{\Lambda}}{\alpha}-\nabla \times \mathbf{u}\right)\right\} \mathrm{d} V \\
& +\int_{S_{\mathrm{f}}} \bar{p} u_{\mathrm{n}}^{S} \mathrm{~d} S
\end{aligned}
$$

where the variables are $p, \mathbf{u}, \boldsymbol{\Lambda}$, and the Lagrange multipliers $\lambda_{p}$ and $\lambda_{\boldsymbol{\Lambda}}$. In addition to the terms used in the $\mathbf{u} / p$ formulation, the fourth term is included to be able to statically condense out the degrees of freedom of the vorticity moment, and the fifth term represents the constraint of

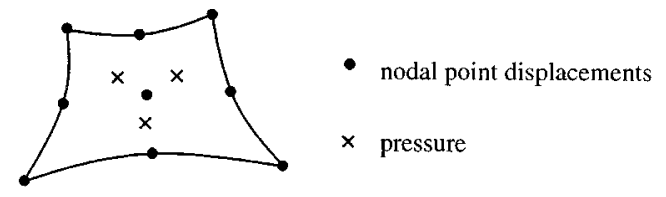

(a)

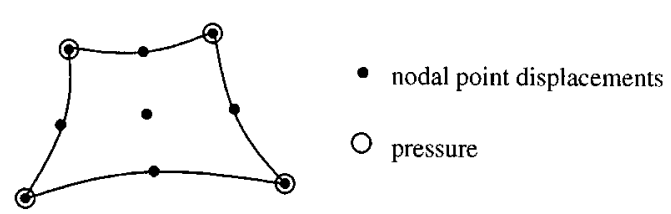

(b)

Figure 1. Two elements for the $\mathbf{u} / p$ formulation. Full numerical integration is used (i.e. $3 \times 3$ Gauss integration): (a) $9 / 3$ element, continuous displacements and discontinuous pressure; (b) $9 / 4-c$ element, continuous displacements and continuous pressure 
equation (23). For the fourth and fifth terms we require that the constant $\alpha$ is large, and we use $\alpha=1000 \beta$. However, from our numerical tests, we find that $\alpha$ can be any numerically reasonable value larger than $\beta$, say $100 \beta \leqslant \alpha \leqslant 10^{6} \beta$.

Invoking the stationarity of $\Pi$, we identify the Lagrange multipliers $\lambda_{p}$ and $\lambda_{\Lambda}$ to be the pressure $p$ and vorticity moment $\Lambda$, respectively, and we obtain the field equations

$$
\begin{array}{r}
\nabla p-\mathbf{f}^{\mathrm{B}}+\nabla \times \boldsymbol{\Lambda}=\mathbf{0} \\
\nabla \cdot \mathbf{u}+p / \beta=0 \\
\nabla \times \mathbf{u}-\boldsymbol{\Lambda} / \alpha=\mathbf{0}
\end{array}
$$

with the boundary conditions

$$
\begin{aligned}
& \mathbf{u} \cdot \mathbf{n}=\bar{u}_{\mathrm{n}} \quad \text { on } S_{u} \\
& p=\bar{p} \quad \text { on } S_{\mathrm{f}} \\
& \boldsymbol{\Lambda}=\mathbf{0} \quad \text { on } S
\end{aligned}
$$

Using the standard Galerkin finite element discretization we now have for a typical element the additional interpolations

$$
\begin{aligned}
\boldsymbol{\Lambda} & =\mathbf{H}_{\Lambda} \hat{\boldsymbol{\Lambda}} \\
\nabla \times \mathbf{u} & =(\nabla \times \mathbf{H}) \hat{\mathbf{U}}=\mathbf{D} \hat{\mathbf{U}}
\end{aligned}
$$

where $\mathbf{H}_{\Lambda}$ is the interpolation matrix, and $\hat{\Lambda}$ is a vector of the unknown vorticity moment values.

The matrix equations of the $\mathbf{u}-p-\boldsymbol{\Lambda}$ formulation are given as

$$
\left[\begin{array}{ccc}
\mathbf{M} & \mathbf{0} & \mathbf{0} \\
\mathbf{0} & \mathbf{0} & \mathbf{0} \\
\mathbf{0} & \mathbf{0} & \mathbf{0}
\end{array}\right]\left\{\begin{array}{c}
\ddot{\hat{\mathbf{U}}} \\
\ddot{\hat{\mathbf{P}}} \\
\ddot{\hat{\mathbf{\Lambda}}}
\end{array}\right\}+\left[\begin{array}{ccc}
\mathbf{0} & \mathbf{L} & \mathbf{Q} \\
\mathbf{L}^{\mathrm{T}} & \mathbf{A} & \mathbf{0} \\
\mathbf{Q}^{\mathrm{T}} & \mathbf{0} & \mathbf{G}
\end{array}\right]\left\{\begin{array}{c}
\hat{\mathbf{U}} \\
\hat{\mathbf{P}} \\
\hat{\boldsymbol{\Lambda}}
\end{array}\right\}=\left\{\begin{array}{c}
\mathbf{R} \\
\mathbf{0} \\
\mathbf{0}
\end{array}\right\}
$$

where

$$
\begin{array}{rlrl}
\mathbf{M} & =\int_{V_{\mathrm{f}}} \rho \mathbf{H}^{\mathrm{T}} \mathbf{H} \mathrm{d} V, & \mathbf{L}=-\int_{V_{\mathrm{f}}} \mathbf{B}^{\mathrm{T}} \mathbf{H}_{p} \mathrm{~d} V \\
\mathbf{Q}=\int_{V_{\mathrm{f}}} \mathbf{D}^{\mathrm{T}} \mathbf{H}_{\Lambda} \mathrm{d} V, & \mathbf{A}=-\int_{V_{\mathrm{f}}} \frac{1}{\beta} \mathbf{H}_{p}^{\mathrm{T}} \mathbf{H}_{p} \mathrm{~d} V \\
\mathbf{G}=-\int_{V_{\mathrm{f}}} \frac{1}{\alpha} \mathbf{H}_{\Lambda}^{\mathrm{T}} \mathbf{H}_{\Lambda} \mathrm{d} V, & \mathbf{R}=-\int_{S_{\mathrm{f}}} \mathbf{H}_{\mathrm{n}}^{S^{\mathrm{T}}} \bar{p} \mathrm{~d} S
\end{array}
$$

Again, the key to the success of the finite element discretization is to choose appropriate interpolations for the displacements, pressure and vorticity moment. Based on the experience with the $\mathbf{u} / p$ formulation, we use the displacement/pressure interpolations that satisfy the inf-sup condition in the analysis of solids (and viscous fluids) and use for the vorticity moment the same or a lower-order interpolation as for the pressure. Thus, some proposed elements for twodimensional analysis are the 9-3-3, 9-3-1 and 9-4c-4c elements. ${ }^{21}$ These elements have displayed good predictive capabilities. However, while we can easily show that the 9-3-1 element satisfies the analytical inf-sup condition, ${ }^{16}$ we could only show that the numerical inf-sup test of Reference 18 is passed for the $9-3-3$ and $9-4 c-4 c$ elements. ${ }^{20}$ Of course, additional elements could be proposed. ${ }^{20,21}$ 


\section{RELATION BETWEEN $\mathbf{u} / p$ AND $\mathbf{u}-p-\boldsymbol{\Lambda}$ FORMULATIONS}

If we compare equation (26) with equation (27), it is clear that the frequencies of the 'vorticity modes' depend on $\alpha$ in the same way as the frequencies of the 'pressure modes' depend on $\beta$. Since $\alpha$ is a numerically large value $\left(100 \beta \leqslant \alpha \leqslant 10^{6} \beta\right)$ in the $\mathbf{u}-p-\boldsymbol{\Lambda}$ formulation, we in fact shift the frequencies of the rotational modes to very high values. Correspondingly, the number of zero frequency modes is reduced by the number of discrete vorticity moment unknowns.

If we substitute equation (27) into equation (25), and use

$$
\nabla(\nabla \cdot \mathbf{u}) \equiv \nabla^{2} \mathbf{u}+\nabla \times(\nabla \times \mathbf{u})
$$

we obtain

$$
\begin{gathered}
\left(1-\frac{\alpha}{\beta}\right) \nabla p-\mathbf{f}^{\mathrm{B}}=\alpha \nabla^{2} \mathbf{u} \\
\nabla \cdot \mathbf{u}+\frac{p}{\beta}=0
\end{gathered}
$$

Therefore, as we assign $\alpha$ a very small value and much smaller than $1 / \beta$, equation (31) approaches equation (9). Hence, while in general we would use $\alpha$ to be a numerically large value in the $\mathbf{u}-p-\boldsymbol{\Lambda}$ formulation, we recognize that by assigning a numerically small value to $\alpha$, the $\mathbf{u} / p$ formulation is obtained.

\section{BOUNDARY CONDITIONS}

Considering the solution of actual fluid flows and fluid flows with structural interactions (in which the fluid is modelled using the Navier-Stokes equations including wall turbulence effects or the Euler equations), we are accustomed to choosing the directions of nodal tangential velocities such that in the finite element discretization there is no transport of fluid across the fluid-structure interfaces. It is important that we employ the same concept also in the definition of the directions of nodal tangential displacements on the boundaries of the acoustic fluid considered here.

To establish the appropriate tangential directions at the typical nodes A and B of our nine-node elements in Figure 2, we need to have

$$
\begin{array}{r}
\int_{L_{\mathrm{a}}} \mathbf{u}_{\mathrm{a}}^{\mathrm{A}} \cdot \mathbf{n}_{\mathrm{a}} \mathrm{d} l+\int_{L_{\mathrm{b}}} \mathbf{u}_{\mathrm{b}}^{\mathrm{A}} \cdot \mathbf{n}_{\mathrm{b}} \mathrm{d} l=0 \\
\int_{L_{\mathrm{a}}} \mathbf{u}_{\mathrm{a}}^{\mathrm{B}} \cdot \mathbf{n}_{\mathrm{a}} \mathrm{d} l=0
\end{array}
$$

where

$$
\begin{array}{rlrl}
\mathbf{n}_{\mathrm{a}} \mathrm{d} l & =\left(-\frac{\partial y_{\mathrm{a}}}{\partial s} \mathrm{~d} s, \frac{\partial x_{\mathrm{a}}}{\partial s} \mathrm{~d} s\right), & \mathbf{n}_{\mathrm{b}} \mathrm{d} l=\left(-\frac{\partial y_{\mathrm{b}}}{\partial s} \mathrm{~d} s, \frac{\partial x_{\mathrm{b}}}{\partial s} \mathrm{~d} s\right) \\
\mathbf{u}_{\mathrm{a}}^{\mathrm{A}} & =\frac{s^{2}+s}{2} \mathbf{U}_{\mathrm{A}}, & \mathbf{u}_{\mathrm{b}}^{\mathrm{A}}=\frac{s^{2}-s}{2} \mathbf{U}_{\mathrm{A}} \\
\mathbf{u}_{\mathrm{a}}^{\mathrm{B}} & =\left(1-s^{2}\right) \mathbf{U}_{\mathbf{B}} & &
\end{array}
$$




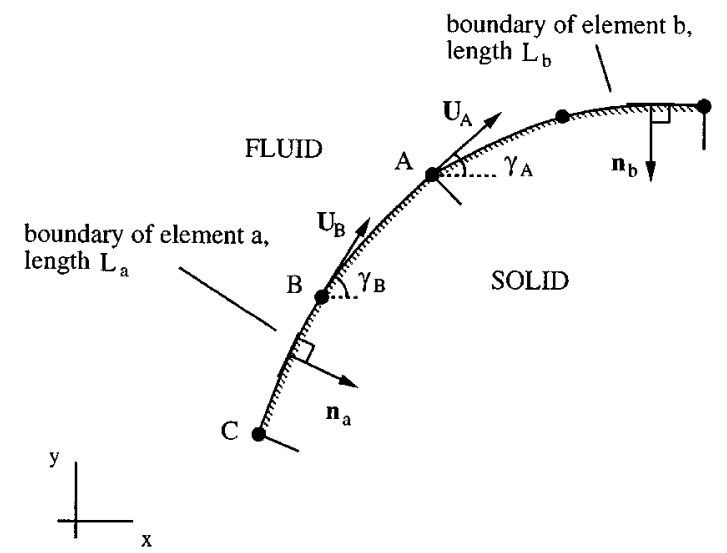

Figure 2. Tangential directions at nodes $A$ and $B$ for nine-node elements

In the above equations, $x_{\mathrm{a}}, y_{\mathrm{a}}, x_{\mathrm{b}}$ and $y_{\mathrm{b}}$ are the interpolated co-ordinates on the boundaries of elements a and $\mathrm{b}$, while $\mathbf{u}_{\mathrm{a}}^{\mathrm{A}}, \mathbf{u}_{\mathrm{b}}^{\mathrm{A}}$ and $\mathbf{u}_{\mathrm{a}}^{\mathrm{B}}$ stand for the interpolated displacements corresponding to the displacement $\mathbf{U}_{\mathrm{A}}$ at node $\mathrm{A}$ and $\mathbf{U}_{\mathrm{B}}$ at node B. Relation (34) implies that the appropriate tangential direction $\gamma_{\mathrm{B}}$ at node $\mathrm{B}$ is given by

$$
\tan \gamma_{\mathrm{B}}=\frac{\partial y}{\partial s} / \frac{\partial x}{\partial s}
$$

Our numerical experiments have shown that it is important to allocate the appropriate tangential directions at all boundary nodes. ${ }^{21}$ Otherwise, spurious non-zero energy modes are obtained in the finite element solution.

\section{EXAMPLE SOLUTIONS}

To demonstrate the capabilities of the proposed $\mathbf{u} / p$ formulation, we present the results of some generic test problems of fluids and fluid-structure interactions. Numerical results of the $\mathbf{u}-p-\boldsymbol{\Lambda}$ formulation have been given in Reference 21.

We set the tall water column and rigid cavity problems to be the same as used in References 6 and 8 in order to compare our results with the results reported using the pure displacementbased formulation and the displacement formulation with the penalty irrotationality constraint.

In all test problems, we want to evaluate the lowest frequencies of the complete system. Of course, we do not calculate any zero frequencies and their mode shapes, but simply shift to the non-zero frequencies sought. ${ }^{16}$

In all cases, the results are found to be in good agreement with those calculated with a displacement-velocity potential $(\mathbf{u}-\phi)$ formulation ${ }^{11}$ or analytical solutions; and the number of zero frequency modes is always exactly equal to the mathematical prediction. Also, in no case was a spurious non-zero frequency predicted. The pressure bands as an error measure are quite smooth indicating accurate solutions. ${ }^{16}$ 


\subsection{Tall water column problem}

Figure 3 shows the tall water column considered in Reference 8. Using the acoustic wave speed $c=\sqrt{\beta / \rho}$ the governing equations for the fluid are

$$
\begin{aligned}
& \nabla^{2} p=\frac{1}{c^{2}} \frac{\partial^{2} p}{\partial t^{2}} \\
& \frac{\partial^{2} \mathbf{u}}{\partial t^{2}}=-\frac{1}{\rho} \nabla p
\end{aligned}
$$

with the initial condition

$$
\mathbf{u}=\mathbf{0} \quad \text { at } t=0
$$

and the boundary conditions

$$
\begin{array}{ll}
u_{1}=0 & \text { at } x_{1}=0, L_{1} \\
u_{2}=0 & \text { at } x_{2}=0 \\
p=0 & \text { at } x_{2}=L_{2}
\end{array}
$$

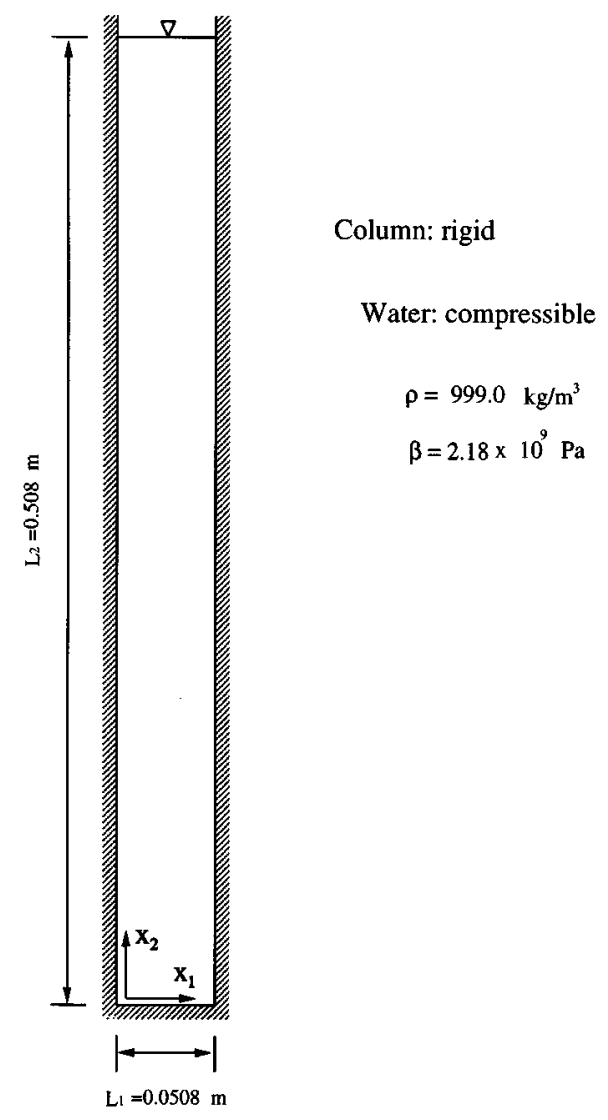

Figure 3. Tall water column problem 
The analytical solution of the above equations is

$$
\begin{aligned}
u_{1} & =A \lambda_{1} \sin (\lambda c t) \cos \left(\lambda_{2} x_{2}\right) \sin \left(\lambda_{1} x_{1}\right) \\
u_{2} & =A \lambda_{2} \sin (\lambda c t) \sin \left(\lambda_{2} x_{2}\right) \cos \left(\lambda_{1} x_{1}\right) \\
p & =-\rho \lambda^{2} c^{2} A \sin (\lambda c t) \cos \left(\lambda_{2} x_{2}\right) \cos \left(\lambda_{1} x_{1}\right)
\end{aligned}
$$

with

$$
\begin{aligned}
\lambda_{1} & =\frac{n \pi}{L_{1}}, \quad n=0,1,2, \ldots \\
\lambda_{2} & =\frac{m \pi}{2 L_{2}}, \quad m=1,3,5, \ldots \\
\lambda^{2} & =\lambda_{1}^{2}+\lambda_{2}^{2} \\
\omega & =c \lambda
\end{aligned}
$$

Here, with no gravity effects, the free surface condition is $p=0$. The calculated lowest four displacement modes and the corresponding pressure distributions are shown in Figures 4 and 5 . The numerical solutions of the frequencies are compared with the analytical values in Table I.

\subsection{Rigid cavity problem}

In Reference 6, Hamdi et al. tested their solution method in the analysis of the rigid cavity problem shown in Figure 6. The analytical frequency solution is

$$
\omega=c \pi \sqrt{\left(\frac{n}{a}\right)^{2}+\left(\frac{m}{b}\right)^{2}}
$$

where $n, m$ are integers and $c$ is the acoustic wave speed.

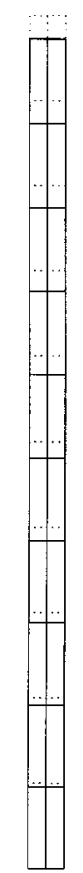

(a)

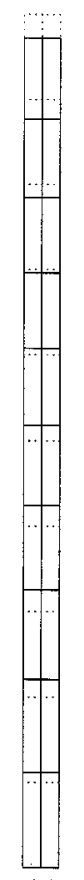

(b)

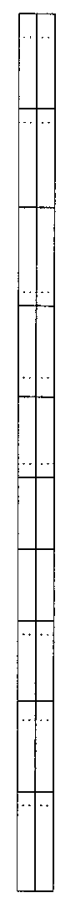

(c)

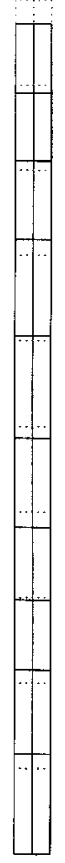

(d)

Figure 4. First four modes of the tall water column problem: (a) Mode 1; (b) Mode 2; (c) Mode 3; (d) Mode 4 


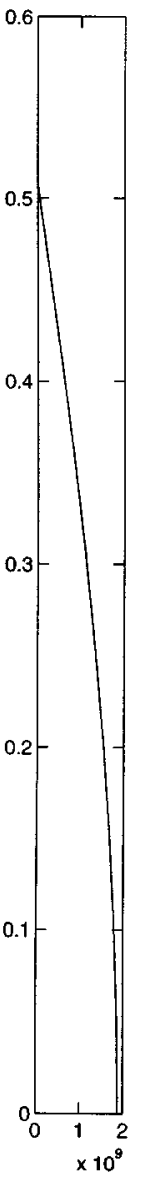

(a)

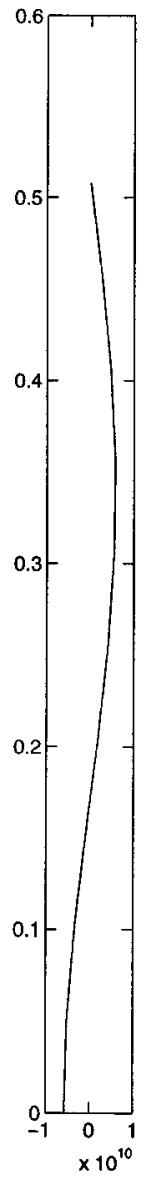

(b)

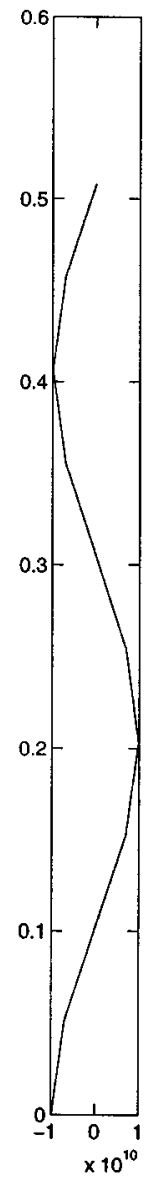

(c)

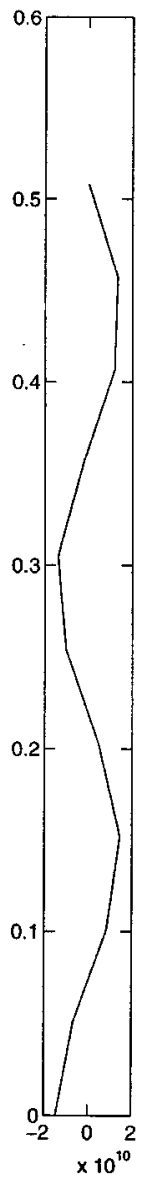

(d)

Figure 5. Pressure distributions corresponding to the first four modes of the tall water column problem $(2 \times 10 \mathrm{mesh}$ of 9/4 - c elements is used): (a) Mode 1; (b) Mode 2; (c) Mode 3; (d) Mode 4

Table I. Results in the tall water column problem using the $\mathbf{u} / p$ formulation

\begin{tabular}{lcccc}
\hline \multirow{2}{*}{$\begin{array}{l}\text { Mixed elements } \\
\text { (mesh of } 2 \times 10 \text { elements) }\end{array}$} & First & Second & Third & Fourth \\
\cline { 2 - 5 } & $4567 \cdot 77$ & $13709 \cdot 8$ & $22915 \cdot 4$ & $32332 \cdot 9$ \\
$9 / 4-c$ & $4567 \cdot 74$ & $13703 \cdot 7$ & $22844 \cdot 6$ & $32004 \cdot 8$ \\
Analytical solution & $4567 \cdot 74$ & $13703 \cdot 2$ & $22838 \cdot 7$ & $31974 \cdot 2$ \\
\hline
\end{tabular}

Figures 7 and 8 show the mode shapes and the pressure distributions of the first four frequencies calculated with a $3 \times 4$ mesh of $9 / 4-c$ elements. Table II compares the numerical results with the analytical values. 


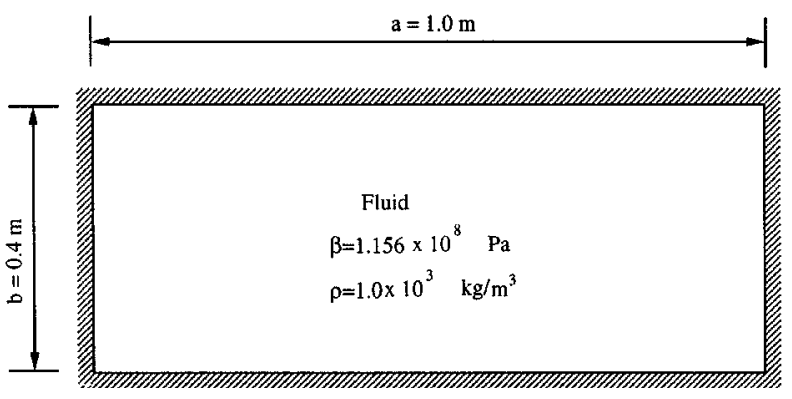

Figure 6. Rigid cavity problem
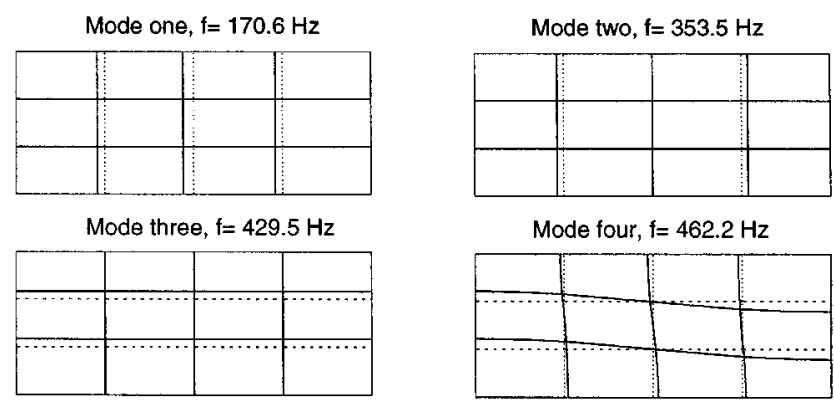

Figure 7. Calculated first four modes in the rigid cavity problem using $3 \times 4$ mesh of $9 / 4-c$ elements

Table II. Results in the rigid cavity problem using the $\mathbf{u} / p$ formulation

\begin{tabular}{lcccc}
\hline & \multicolumn{4}{c}{ Frequencies $(\mathrm{Hz})$} \\
\cline { 2 - 5 } \begin{tabular}{l} 
Mixed elements \\
\cline { 2 - 5 } mesh $)$
\end{tabular} & First & Second & Third & Fourth \\
\hline $9 / 4-c$ & $170 \cdot 6$ & $353 \cdot 5$ & $429 \cdot 5$ & $462 \cdot 2$ \\
$9 / 3$ & $170 \cdot 0$ & $341 \cdot 3$ & $425 \cdot 3$ & $468 \cdot 1$ \\
Analytical solution & $170 \cdot 0$ & $340 \cdot 0$ & $425 \cdot 0$ & $457 \cdot 7$ \\
\hline
\end{tabular}

\subsection{Three paradigms of fluid-structure interactions}

Figure 9 describes the tilted piston-container problem. The massless elastic piston moves horizontally. Figure 10 describes the problem of a rigid cylinder vibrating in an acoustic cavity. The cylinder is suspended from a spring and vibrates vertically in the fluid. Figure 11 shows a rigid ellipse on a spring in the same acoustic cavity.

Tables III and IV list the results obtained using the $\mathbf{u} / p$ formulation with $9 / 3$ elements and using the velocity potential $(\mathbf{u}-\phi)$ formulation. ${ }^{11}$ The meshes used in these analyses have been derived by starting with coarse meshes and subdividing in each refinement each element into two or four elements. 

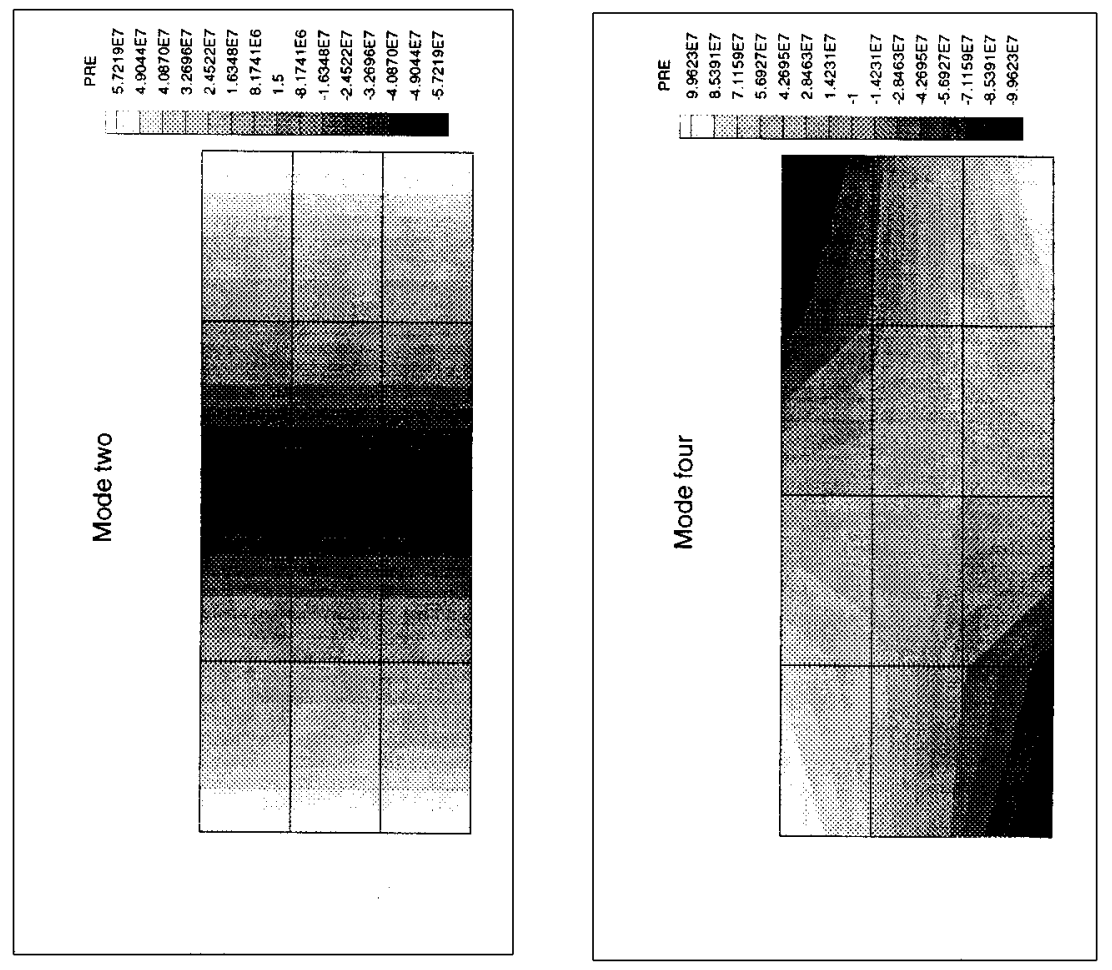

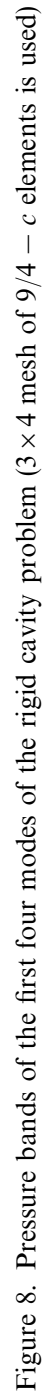

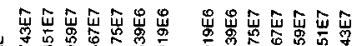

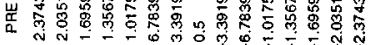
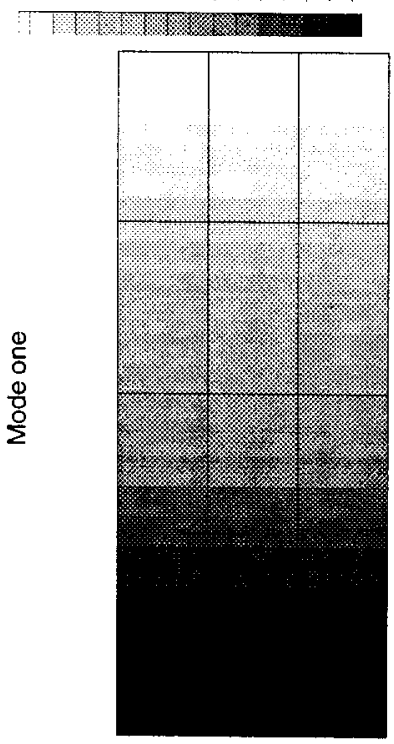

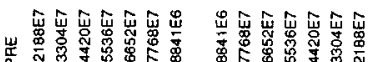

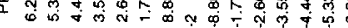

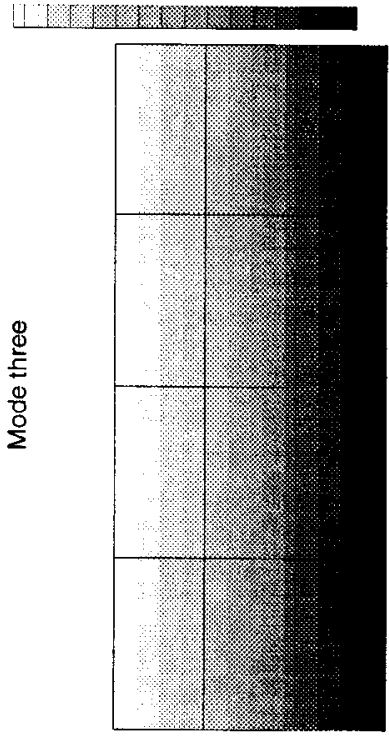




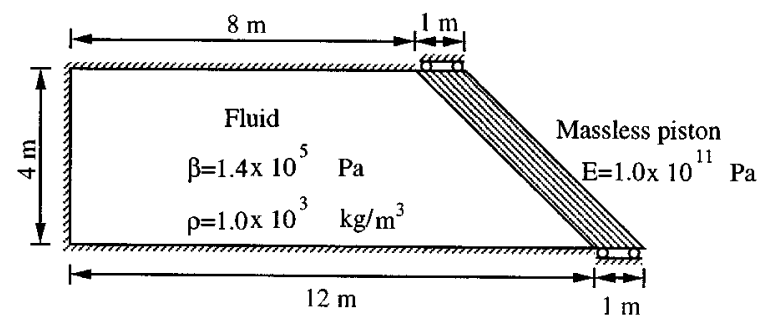

Figure 9. Tilted piston-container system

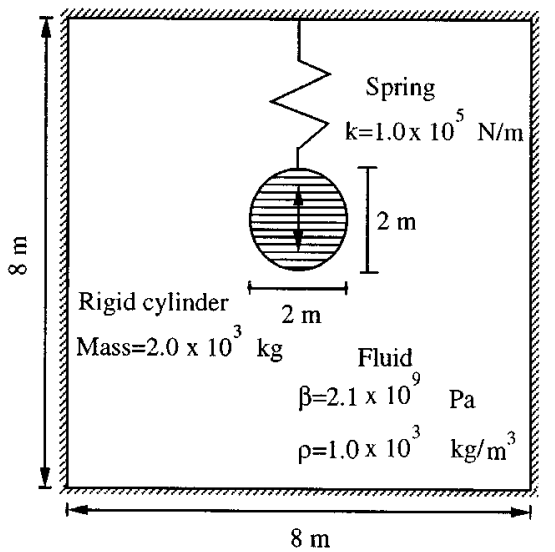

Figure 10. A rigid cylinder vibrating in an acoustic cavity

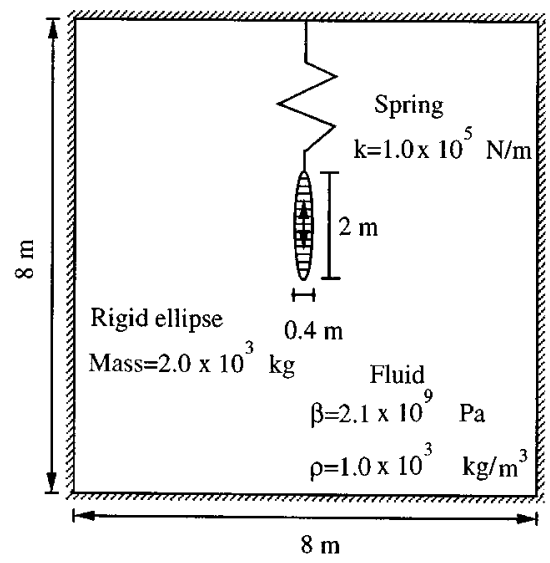

Figure 11. A rigid ellipse vibrating in an acoustic cavity 
Table III. Results in test problems using the $\mathbf{u} / p$ formulation with $9 / 3$ elements

\begin{tabular}{lccccc}
\hline & & \multicolumn{4}{c}{ Frequencies (rad/s) } \\
\cline { 3 - 6 } Test case & $\begin{array}{c}\text { Mesh no. } \\
\text { of elements }\end{array}$ & First & Second & Third & Fourth \\
\hline Tilted & 4 & $1 \cdot 898$ & $6 \cdot 063$ & $9 \cdot 275$ & $10 \cdot 45$ \\
piston- & 16 & $1 \cdot 867$ & $5 \cdot 702$ & $9 \cdot 239$ & $9 \cdot 808$ \\
container & 32 & $1 \cdot 862$ & $5 \cdot 605$ & $9 \cdot 192$ & $9 \cdot 397$ \\
Rigid & 2 & $3 \cdot 899$ & $718 \cdot 2$ & 1178 & 1326 \\
cylinder & 8 & $4 \cdot 259$ & $611 \cdot 5$ & 1193 & 1330 \\
problem & 32 & $4 \cdot 285$ & $589 \cdot 6$ & 1138 & 1254 \\
Rigid & 2 & $6 \cdot 192$ & $697 \cdot 8$ & 1216 & 1269 \\
ellipse & 8 & $6 \cdot 755$ & $591 \cdot 7$ & 1229 & 1235 \\
problem & 32 & $6 \cdot 848$ & $572 \cdot 6$ & 1157 & 1178 \\
\hline
\end{tabular}

Table IV. Results obtained using the $\mathbf{u}-\phi$ formulation for analysis of three test problems

\begin{tabular}{lccccc}
\hline & \multirow{3}{*}{$\begin{array}{c}\text { Mesh no. } \\
\text { Test case }\end{array}$} & \multicolumn{4}{c}{ Frequencies (rad/s) } \\
\cline { 3 - 6 } & of elements & First & Second & Third & Fourth \\
\hline Tilted piston problem & 32 & $1 \cdot 858$ & $5 \cdot 569$ & $9 \cdot 116$ & $9 \cdot 299$ \\
Rigid cylinder problem & 32 & $4 \cdot 269$ & $581 \cdot 8$ & 1124 & 1224 \\
Rigid ellipse problem & 32 & $7 \cdot 071$ & $563 \cdot 2$ & 1138 & 1158 \\
\hline
\end{tabular}

\section{CONCLUSIONS}

In this paper we have presented an effective displacement/pressure finite element formulation for the analysis of acoustic fluid-structure interaction problems. In the $\mathbf{u} / p$ formulation we interpolate displacements and pressure as independent variables, and we employ elements that satisfy the inf-sup condition. If a discontinuous pressure approximation is used (such as for the $9 / 3$ element), the pressure degrees of freedom are statically condensed out on the element level, so that only the nodal displacement degrees of freedom (also employed for the solid elements) are present in the assemblage process. ${ }^{16}$ It is also important that the slip boundary conditions are introduced such that the requirements of mass and momentum conservation around the fluid boundaries and fluid-structure interfaces are satisfied.

We conclude that in frequency analysis, the historically reported non-zero frequency spurious modes were caused by the pure displacement formulation (including the penalty formulations), the use of mixed elements which do not satisfy the inf-sup condition and the improper treatment of the boundary conditions.

Using the $\mathbf{u} / p$ formulation for the solution of frequencies, many (exact) zero frequencies are obtained. The exact number of these zero frequencies can be predicted, and in the solution the eigensolver simply shifts over them. ${ }^{16}$ Also, the number of zero frequencies can be reduced by use of the $\mathbf{u}-p-\boldsymbol{\Lambda}$ formulation (see also Reference 21 ). 
While we did not specifically consider the effects of gravity and large fluid motions, an advantage of the formulation given is that these effects can directly be included as in the analysis of solids. ${ }^{16}$

\section{REFERENCES}

1. O. C. Zienkiewicz and P. Bettess, 'Fluid-structure dynamics interaction and wave forces. An introduction to numerical treatment', Int. j. numer. methods eng., 13, 1-16 (1978).

2. T. Belytschko, 'Fluid-structure interaction', Comput. Struct., 12, 459-469 (1980).

3. M. K. Hakala, 'Application of the finite element method to fluid-structure interaction in ship vibration', Research Report, 433, Technical Research Center of Finland, 1986.

4. C. A. Felippa and R. Ohayon, 'Mixed variational formulation of finite element analysis of acoustoelastic/slosh fluid-structure interaction', J. fluids struct., 4, 35-57 (1990).

5. K. J. Bathe and W. F. Hahn, 'On transient analysis of fluid-structure systems', Comput. Struct., 10, 383-391 (1979).

6. M. A. Hamdi, Y. Ousset and G. Verchery, 'A displacement method for the analysis of vibrations of coupled fluid-structure systems', Int. j. numer. methods eng., 13, 139-150 (1978).

7. T. B. Belytschko and J. M. Kennedy, 'A fluid-structure finite element method for the analysis of reactor safety problems', Nucl. Eng. Des., 38, 71-81 (1976).

8. L. G. Olson and K. J. Bathe, 'A study of displacement-based fluid finite elements for calculating frequencies of fluid and fluid-structure systems', Nucl. Eng. Des., 76, 137-151 (1983).

9. H. Morand and R. Ohayon, Fluid-Structure Interaction. Wiley, New York, 1995. Translated by C. A. James.

10. G. C. Everstine, 'A symmetric potential formulation for fluid-structure interaction', J. Sound Vib., 79(1), 157-160 (1981).

11. L. G. Olson and K. J. Bathe, 'Analysis of fluid-structure interactions. A direct symmetric coupled formulation based on the fluid velocity potential', Comput. Struct., 21, 21-32 (1985).

12. L. Kiefling and G. C. Feng, 'Fluid-structure finite element vibrational analysis', AIAA J., 14(2), 199-203 (1976).

13. E. L. Wilson and M. Khalvati, 'Finite elements for the dynamic analysis of fluid-solid systems', Int. j. numer. methods eng., 19, 1657-1668 (1983).

14. H. C. Chen and R. L. Taylor, 'Vibration analysis for fluid-solid systems using a finite element displacement formulation', Int. j. numer. methods eng., 29, 683-698 (1990).

15. A. Bermúdez and R. Rodríguez, 'Finite element computation of the vibration modes of a fluid-solid system', Comput. Methods Appl. Mech. Eng., 119, 355-370 (1994).

16. K. J. Bathe, Finite Element Procedures, Prentice Hall, Englewood Cliffs, N.J., 1996

17. F. Brezzi and K. J. Bathe, 'A discourse on the stability conditions for mixed finite element formulations', Comput. Methods Appl. Mech. Eng., 82, 27-57 (1990).

18. D. Chapelle and K. J. Bathe, 'The inf-sup test', Comput. Struct., 47, 537-545 (1993).

19. D. Pantuso and K. J. Bathe, 'A four-node quadrilateral mixed-interpolated element for solids and fluids', Math. Models \& Methods Appl. Sci., 5, 1113-1128 (1995).

20. X. Wang and K. J. Bathe, 'On mixed elements for acoustic fluid-structure interactions', Math. Models \& Methods Appl. Sci., In press.

21. K. J. Bathe, C. Nitikitpaiboon and X. Wang, 'A mixed displacement-based finite element formulation for acoustic fluid-structure interaction', Comput. Struct., 56, 225-237 (1995). 